\title{
Present situation and development of wind power generation and its
}

\section{forecasting technology}

\author{
Wei Tian ${ }^{1, a}$, Jingyi Zhou ${ }^{1, b}$ and Hui Li ${ }^{1, c}$ \\ ${ }^{1}$ College of Metallurgy and Energy, North China University of Science and \\ Technology, Tangshan Hebei 063009, China \\ atwf@ncst.edu.cn, 'bhoujingyi@ncst.edu.cn, 'Th@ncst.edu.cn
}

Key words: Wind power generation, wind power forecast, research direction, enterprise strategy Abstract: Because wind energy is very rich, the price is very cheap, energy will not be exhausted, and can be obtained in a wide range, very clean, no pollution, will not affect the climate, so wind power has been the world's fastest growing energy. This paper summarizes and tracks the main forecasting techniques of wind power generation in the past 10 years, and describes the power generation forecasting scheme based on statistics and artificial intelligence technology. This paper points out the shortcomings and improvement direction of various forecasting techniques, analyzes the present situation of wind power generation at home and abroad, expounds the development trend of wind power generation, and gives out the Countermeasures for the future of our country.

\section{Introduction}

Development of renewable resources is the main way to solve the energy crisis, renewable energy generation is efficient and clean energy use, only for wind power, the wind power generation will meet $12 \%$ of the world's electricity demand in 2020 . Wind power has been the world's fastest growing energy, in the past 10 years, the world wind power plant in the $30 \%$ annual growth rate in the development. Because of the obvious advantages of wind power generation, the world is competing for investment, research and development of new wind power equipment

\section{Present situation and development of wind power forecasting technology}

Wind power generation forecasting is composed of three parts, including the wind speed forecast ${ }^{[1]}$, the power curve calculation and the model output statistics. But because of the influence of temperature, pressure difference, air density and terrain, the wind speed is one of the most difficult weather parameters.

All wind power generation forecasting models include two stages: (1) data collection; 2) data processing. The role of data collection is to provide input information to the forecast model. The most important data is the historical data of the meteorological parameters and wind power generation capacity. The sources of data collection include the weather forecast information of the measurement apparatus (e.g., weather sensors, etc.) and weather departments deployed around the wind farm.

\section{Indirect forecasting method for wind power generation}

As the wind power generation is mainly determined by the wind speed, the majority of the wind power generation forecasting model is the wind speed forecast, and then use the power curve of the power generation unit to convert the wind speed forecast to the power generation capacity forecast value. In the wind speed forecast, there are four basic models: physical forecasting model; based on 
the statistical forecasting model; the forecast model based on artificial intelligence technology; combination forecasting model.

\section{Physical prediction}

The physical model based on numerical weather prediction is a traditional wind speed forecasting technique, which is similar to the general weather forecast. The main idea is to calculate the wind speed by using the numerical method on the computer.

\section{Prediction based on statistics}

The idea of statistical forecasting model is based on the historical information of wind speed time series data observed by the system, and the model is built by curve fitting and parameter estimation. Compared with other forecasting models, the statistical model is more effective than other forecasting models, but because of the statistical properties (such as auto correlation and standard deviation) of wind speed time series data, the forecasting error will be increased with the increase of forecast time.

\section{Forecast based on artificial intelligence technology}

The essence of artificial intelligence is to simulate the process of human thinking. The forecasting model based on artificial intelligence technology has the ability to learn, and the prediction accuracy can be improved with the increase of historical data. Compared with the statistical forecasting model, artificial intelligence prediction model is more easy to build and the required modeling time is too short, it does not need to determine the mathematical expression between the wind speed input and output, but by the training of the forecasting model to get the forecast value.

\section{Combined forecasting}

The combination forecasting is obtained by comparing the forecast values of different forecasting schemes to obtain an optimal statistical forecasting model. The basic idea is that if there are errors in the prediction value of different forecasting methods, there is no deviation, and there is a low correlation between each other. The combination forecasting method is highly related to the combination of the advantages, so in the construction of combination forecasting, the basic prediction scheme of the original observation data and input data must be significantly different.

\section{Conclusions}

Has important significance to the wind power generation forecast. The following suggestions are put forward: (1) in wind power generation forecasting, an important reason is that the indirect prediction model is a key reason is that the wind speed forecast value directly through the wind power generation unit manufacturers to provide the power curve, but it is the power of the manufacturer to obtain the power curve, so it can not accurately describe the relationship between wind speed and power generation in various conditions. In order to further improve the prediction accuracy, we need to get a more precise relationship based on the actual situation, the use of relevant technologies such as statistical methods, etc., (2) the accuracy of all the prediction models are dependent on the accuracy of the original input data, and can be used to collect these meteorological information through the deployment of remote gas sensors.

\section{Present situation and development of wind power generation}

\section{Current situation of wind power generation industry in foreign countries}

In the last 30 years, the world has made great progress in the utilization of wind energy, both in theory and in application. Today, the wind power in Europe has been able to meet the needs of 40000000 people's lives. The European Wind Energy Association is expected to have nearly two 
hundred million people in 2020, the total use of wind power, accounting for half of the European population.

\section{Current situation of domestic wind power generation}

In China, for a long time, people take the wind power electricity price is higher than the electricity price, which has been neglected in the wind power as a clean energy for energy shortage and environmental protection ${ }^{[2]}$.

In policy, the lack of wind power development goals and the implementation of strategic planning; two is the lack of effective economic incentive policies and strong institutional safeguards; three is the lack of incentives for wind turbine localization policy measures; four is the lack of effective investment and financing system, the lack of government guidance under the wind power purchase policy, private enterprise investment in wind power Internet is still institutional barriers; six is the lack of strong publicity, public awareness of renewable resources.

Second, the technical assessment of the full scale of the wind power, the wind power is not scientific assessment of the feasibility of the wind power generation is relatively concentrated, there is a large gap between the wind power generation, there is a large gap between Inner Mongolia, northwest, and coastal areas, three is China's wind resources are mainly concentrated in, Northwest China, and coastal areas, the presence of wind power and energy storage problems, the wind power and energy storage problems, the existence of wind power and energy storage problems, the wind power and energy storage problems, the wind power and energy storage problems, the wind power and energy storage problems, the wind power and energy storage problems, the wind power and energy storage problems, the wind power and energy storage problems, the wind power generation $^{[3]}$.

\section{The development trend of wind power generation in China and enterprise strategy}

The development trend of wind power generation, the first is the rapid decline of the wind power, has become increasingly close to the cost of coal, economic benefits. Two is the construction period is short, quick, and effective, thermal power, water and electricity construction period need to be calculated in years, o Three is to curb the development of greenhouse effect, and vigorously develop wind energy can greatly reduce the carbon dioxide caused by greenhouse effect, to ease the situation of climate warming, can effectively curb the sandstorm disaster, and inhibit the development of desertification.

In China, enterprises should actively follow up the world advanced technology, and constantly promote technological progress, reduce costs, establish efficient sales system, in the quality, price and service to form their core competitiveness, in technology, research and development, management and production efficiency to achieve first-class level. Accelerate R \& D technology transformation and application ${ }^{[4]}$, to seize the market's real needs, fast new marketable products put on the market in order to make the company have faster development.

\section{Conclusions}

In summary, with the continuous improvement of the market economy system and the continuous improvement of wind power industry laws and regulations, the wind power generation enterprises will face more fierce international competition market, and wind power generation enterprises should make and implement the correct development strategy and objectives, adjust product structure, optimize resource allocation, promote enterprise product innovation, improve enterprise's market competitiveness. 


\section{Acknowledgements}

This work was financially supported by the National Natural Science Foundation of China (Grant No. 51401075)

\section{Reference}

[1]DeFu Li: 2005 small wind power industry status and development of wind energy in China[J]. China wind energy. Vol.2 (2006),p. 9- 11.In Chinese

[2]GenYing Yan, Yibin Xiao. Analysis of the current situation and development trend of wind power generation[J]. Shopping malls modernization.(2008),p.215-216. In Chinese

[3]Yuanyi Chen,Zengwei Zheng, Meimei Huo, et al. The present situation and development of wind power forecasting technology research [J].Energy engineering. Vol.6(2012),p.18 21. In Chinese

[4]Dexin He. 2020 China's science and technology development research [J].Technology and industry.Vol.1 (2004),4,p. 36-37. In Chinese 